\title{
General static load capacity in four contact point slewing bearings
}

\author{
J. Aguirrebeitia, R. Avilés, I. F. de Bustos \& M. Abasolo \\ Department of Mechanical Engineering, \\ University of the Basque Country, Spain
}

\begin{abstract}
This work tries to represent the general static load-carrying capacity of fourcontact-point slewing bearings under general loading. This representation is based on a generalization of Sjoväll and Rumbarger's equations and provides an acceptance surface in the load space. This acceptance surface provides the key set of acceptance curves for the design and selection of bearings.
\end{abstract}

Keywords: four contact point slewing bearing, acceptance surface, bearing selection, static load capacity.

\section{Introduction}

Slewing bearings are large-sized bearings with many applications, such as in wind turbine generators, tower cranes vertical lathe tables... The loads acting on these bearings usually contain axial and radial forces, as well as tilting moments (see Figure 1). In the most unfavourable load case, the radial force is perpendicular to the resultant of the tilting moments. Several bearing manufacturers provide acceptance curves that allow one to determine whether or not a bearing is acceptable for a given equivalent load, calculated as a combination of the axial and radial loads. By means of a moment-axial-force diagram, this equivalent load allows a designer to obtain the maximum allowable tilting moment that the bearing can bear. This is illustrated in Figure 2. There are some variations in the form and limits of the diagram shown in Figure 2. depending on the manufacturers having experimented with or assessed the bearings themselves, or having simply copied other manufacturers' data. Anyhow, there is always a certain ambiguity and a lack of a clear criterion in the 
definition of the equivalent load. The objective of this work is the development of a procedure which defines a surface by the limiting values of the loads $F_{\mathrm{A}}, F_{\mathrm{R}}$ and $M$ for four-contact-point slewing bearings. This representation can be used directly to determine whether or not a given load combination is acceptable.

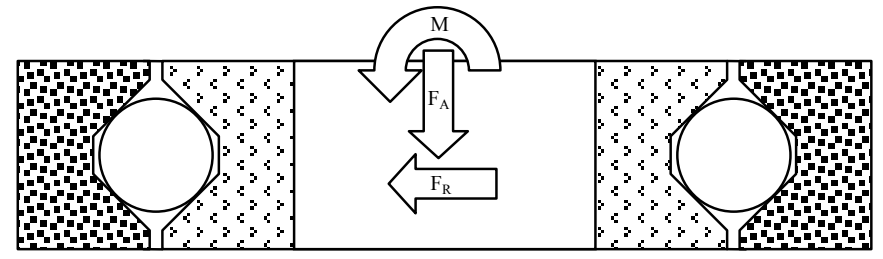

Figure 1: Loads acting on a slewing bearing.

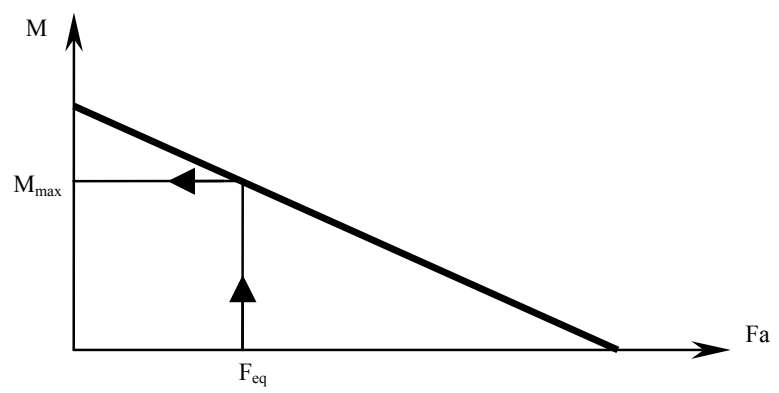

Figure 2: Moment-axial-force diagram for a four-contact-point slewing bearing.

There have been some previous publications where concepts relevant to the assessment of the static load-carrying capacity of four-contact-point slewing bearings have been examined [1-5]. All of the above papers propose a generalization of the equations obtained by Jones [6], in which the load distribution is worked out from the known external loads, taking account of the variation in contact angle with the loading conditions.

This work has a different focus, consisting in directly calculating the load combinations that result in static failure (as defined in the ISO standard [7]) of the most loaded element. This allows one to obtain a three-dimensional acceptance condition in the form of a surface inequation. The designer can use this acceptance surface as a straightforward way to select a bearing appropriately. This approach is based on the calculations of Sjoväll [8] for combinations of axial and radial loads and of Rumbarger [9] for combinations of axial and moment loads. These calculations assume zero clearance in the contact, and rigid rings. These assumptions are also made in the current paper. The axial load-carrying capacity is used to normalize the results and can be obtained from standards [7]. 


\section{The three-dimensional acceptance condition}

As in [8] and [9], the first step consists on arranging a geometrical interference model, and then, taking the interference field as deformation field, equations that reflect the equilibrium of the forces and moments are worked out. Finally, the equilibrium equations are rewritten to provide an acceptance inequation. It has been assumed that only the inner ring can be displaced, while the outer one and the balls remain fixed. The deformation field can be expressed as follows, measured as displacements perpendicular to raceways 1 and 2:

$$
\begin{aligned}
& \delta_{\psi}^{1}=\delta_{a} \sin \alpha+\delta_{r} \cos \alpha \cos \psi+\frac{\theta d}{2} \sin \alpha \cos \psi \\
& \delta_{\psi}^{2}=-\delta_{a} \sin \alpha+\delta_{r} \cos \alpha \cos \psi-\frac{\theta d}{2} \sin \alpha \cos \psi
\end{aligned}
$$

where $\psi$ is the angular location over the rings, $\alpha$ the contact angle, $d$ is the contact diameter and $\delta_{\mathrm{a}}, \delta_{\mathrm{r}}, \theta$ are the axial radial and angular displacements of the inner ring with respect the outer one. Superscript 1 refers to the upper raceway and superscript 2 refers to the lower one.

We define auxiliary variables $A, R$ and $M$ as follows:

$$
\begin{aligned}
& A=\delta_{a} \sin \alpha \\
& R=\delta_{r} \cos \alpha \\
& M=\frac{\theta d}{2} \sin \alpha
\end{aligned}
$$

Using equation (2), equation (1) can be written as:

$$
\begin{aligned}
& \delta_{\psi}^{1}=A+(R+M) \cos \psi \\
& \delta_{\psi}^{2}=-A+(R-M) \cos \psi
\end{aligned}
$$

The maximum and minimum values of the deformation fields occur when $\psi=$ 0 and $\psi=\pi$, so that

$$
\begin{aligned}
& \delta_{0}^{1}=A+R+M \\
& \delta_{\pi}^{1}=A-R-M \\
& \delta_{0}^{2}=-A+R-M \\
& \delta_{\pi}^{2}=-A-R+M
\end{aligned}
$$

If we rewrite equations (12) in terms of the values of the deformation for $\psi=0$ and $\psi=\pi$ : 
20 Tribology and Design

$$
\begin{aligned}
& \delta_{\psi}^{1}=\frac{1}{2}\left[\left(\delta_{0}^{1}+\delta_{\pi}^{1}\right)+\left(\delta_{0}^{1}-\delta_{\pi}^{1}\right) \cos \psi\right] \\
& \delta_{\psi}^{2}=\frac{1}{2}\left[\left(\delta_{0}^{2}+\delta_{\pi}^{2}\right)+\left(\delta_{0}^{2}-\delta_{\pi}^{2}\right) \cos \psi\right]
\end{aligned}
$$

According with expressions developed in [8] and [9], and after some mathematical adaptations, action equilibrium equations can be written as shown below, where contributions of raceways 1 and 2 are considered.

$$
\begin{aligned}
& \frac{F_{A}}{Q_{M A X} Z \sin \alpha}=F_{Q}^{1} J_{I}\left(\delta_{0}^{1}, \delta_{\pi}^{1}\right)-F_{Q}^{2} J_{I}\left(\delta_{0}^{2}, \delta_{\pi}^{2}\right) \\
& \frac{F_{R}}{Q_{M A X} Z \cos \alpha}=F_{Q}^{1} J_{I I}\left(\delta_{0}^{1}, \delta_{\pi}^{1}\right)+F_{Q}^{2} J_{I I}\left(\delta_{0}^{2}, \delta_{\pi}^{2}\right) \\
& \frac{M / d}{Q_{M A X} Z \sin \alpha}=F_{Q}^{1} \frac{1}{2} J_{I I}\left(\delta_{0}^{1}, \delta_{\pi}^{1}\right)-F_{Q}^{2} \frac{1}{2} J_{I I}\left(\delta_{0}^{2}, \delta_{\pi}^{2}\right)
\end{aligned}
$$

where

$$
\begin{aligned}
& J_{I}\left(\delta_{0}, \delta_{\pi}\right)=\frac{1}{2 \pi} \int_{\delta+}\left(\frac{\delta_{\psi}}{\delta_{M A X}}\right)^{\frac{3}{2}} d \psi \\
& J_{I I}\left(\delta_{0}, \delta_{\pi}\right)=\frac{1}{2 \pi} \int_{\delta+}\left(\frac{\delta_{\psi}}{\delta_{M A X}}\right)^{\frac{3}{2}} \cos \psi d \psi
\end{aligned}
$$

and

$$
\begin{aligned}
& \text { if }\left\{\begin{array}{c}
\delta_{0}^{1}=\max \left\{\delta_{0}^{1}, \delta_{\pi}^{1}, \delta_{0}^{2}, \delta_{\pi}^{2}\right\} \\
\text { or } \\
\delta_{\pi}^{1}=\max \left\{\delta_{0}^{1}, \delta_{\pi}^{1}, \delta_{0}^{2}, \delta_{\pi}^{2}\right\}
\end{array}\right\} \text { then }\left\{\begin{array}{c}
F_{Q}^{1}=1 \\
\left.F_{Q}^{2}=\left(\frac{\max \left\{\delta_{0}^{2}, \delta_{\pi}^{2}\right\}}{\max \left\{\delta_{0}^{1}, \delta_{\pi}^{1}\right\}}\right)^{\frac{3}{2}}\right\} \\
\text { or }\left\{\begin{array}{c}
\delta_{0}^{2}=\max \left\{\delta_{0}^{1}, \delta_{\pi}^{1}, \delta_{0}^{2}, \delta_{\pi}^{2}\right\} \\
\delta_{\pi}^{2}=\max \left\{\delta_{0}^{1}, \delta_{\pi}^{1}, \delta_{0}^{2}, \delta_{\pi}^{2}\right\}
\end{array}\right\} \text { then }\left\{\begin{array}{c}
F_{Q}^{1}=\left(\frac{\max \left\{\delta_{0}^{1}, \delta_{\pi}^{1}\right\}}{\max \left\{\delta_{0}^{2}, \delta_{\pi}^{2}\right\}}\right)^{\frac{3}{2}} \\
F_{Q}^{2}=1
\end{array}\right\}
\end{array}\right.
\end{aligned}
$$

\section{Results and discussion}

The maximum load is expressed as a function of the axial load-carrying capacity. This is done in order to represent graphically the values of $F_{A}, F_{R}$ and $M$ that cause permanent deformation in the most loaded ball, as detailed in [7]. We have:

$$
Q_{M A X}=\frac{C_{0 a}}{Z \sin \alpha}
$$


When we substitute equation (9) into equation (6), we obtain:

$$
\begin{aligned}
& \frac{F_{A}}{Q_{M A X} Z \sin \alpha}=\frac{F_{A}}{C_{0 a}} \\
& \frac{F_{R}}{Q_{M A X} Z \cos \alpha}=\frac{F_{R}}{C_{0 a}} \tan \alpha \\
& \frac{M / d}{Q_{M A X} Z \sin \alpha}=\frac{M / d}{C_{0 a}}
\end{aligned}
$$

which can be seen as the coordinates of a point in a $3 \mathrm{D}$ diagram, with axes $F_{A} / C_{0 a},\left(F_{R} / C_{0 a}\right) \tan \alpha$ and $(M / d) / C_{0 a}$. When we study different deformation fields defined by $(A, R, M)$ according to equation (4) and solve equation (6) for each case, the final result is a cloud of points that define the acceptance surface.

It is difficult to represent the acceptance surface graphically, since it must be mapped with the parametric coordinates $(A, R, M)$; each set of parametric coordinates results in another set in the coordinate system $\left(F_{A} / C_{0 a}, F_{R} \tan \alpha / C_{0 a}\right.$, $\left.(M / d) / C_{0 a}\right)$. A MAPLE application has been developed to assess the cloud of points with various levels of accuracy $N$ in order to map the surface, as follows:

$$
\left.\begin{array}{l}
A(i)=i \\
R(i)=i \\
M(i)=i
\end{array}\right\} \quad i=-N \ldots N
$$

This is done in such a way that all possible combinations are chosen, resulting in $(2 N+1)^{3}$ points. Figure 3 shows a rendered triangulation of the cloud of points for $N=20$ (68921 points).

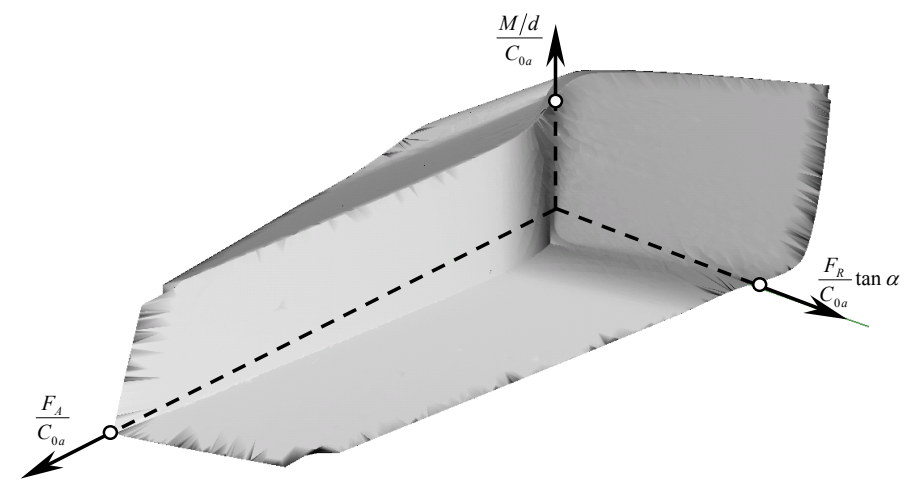

Figure 3: $\quad$ Moment-axial-force diagram for a four-contact-point.

The validity of the method has been verified by, for instance, checking the intersection of the surface with the coordinate axes. In fact, as can be found using 
the results in [10] and [11] applied to a four-contact-point slewing bearing, the limiting values of the axial and radial forces and tilting moment should be determined by

$$
\begin{gathered}
F_{A, \text { max }}=C_{0 a} \\
F_{R, \text { max }}=\frac{2}{4.37} C_{0 a}=0.4577 C_{0 a} \\
M_{\text {max }}=\frac{d}{4.37} C_{0 a}=0.2288 C_{0 a} d
\end{gathered}
$$

These values match up with the ones given by the method developed in this paper. However, if the same comparison is done with intersection curves with the coordinate planes (as in [8] and [9]) it must be pointed out that there are some slight differences. In this sense, the method developed in this paper is more complete than those presented in [8] and [9], and it should not be seen as a superposition of the results of those methods. The reason is that in [8], only axial- and radial-force equilibrium is assumed, whereas the moment generated by axial non-uniform loading is not balanced, and in [9], only axial-force and tilting-moment equilibrium is assumed, whereas the axially generated radial force is not balanced.

\section{Conclusions and future work}

In this work a procedure for the assessment of the limiting values of the loads acting on a four-contact-point slewing bearing has been presented. The loads are an axial force, a tilting moment, and a radial force perpendicular to that moment. These limiting values are obtained by considering the equilibrium of the forces and moments in the inner ring, and then equating the maximum load to the value obtained from the axial load-carrying capacity. This procedure results in a surface expressed as a cloud of points in a three-dimensional coordinate system whose axes are the normalized loads. A designer can use this acceptance surface to provide a straightforward way to select a bearing.

Further work remains to be done, using for example multiparametric NURBS surfaces to assess equivalent loads in order to systematically obtain curves for the selection of bearings.

\section{References}

[1] Amasorrain, J.I., Sagartzazu, X., Damián, J., Load distribution in a four contact-point slewing bearing. Mechanism and Machine Theory, 38, 479496, 2003.

[2] Liao, N.T., Lin, J.F., A new method for the analysis of deformation and load in a ball bearing with variable contact angle. Journal of Mechanical Design, 123, 304-312, 2001.

[3] Zupan, S., Prebil, I., Carrying angle and carrying capacity of a large single row ball bearing as a function of geometry parameters of the rolling contact 
and the supporting structure stiffness. Mechanism and Machine Theory, 36, 1087-1103, 2001.

[4] Antoine, J.F., Abba, G., Molinari, A., A new proposal for explicit angle calculation in angular contact ball bearing. Journal of Mechanical Design, 128, 468-478, 2006.

[5] Hernot, X., Sartor, M., Guillot, J., Calculation of the stiffness matrix of angular contact ball bearings by using the analytical approach. Journal of Mechanical Design, 122, 83-90, 2000.

[6] Jones, A., Analysis of Stresses and Deflections. New Departure Engineering Data, Bristol, CT, 1946.

[7] International Organization for Standardization, Rolling Bearings - Static Load Ratings, ISO 76:2006, 3rd edition, 2006.

[8] Sjoväll, H. The load distribution within ball and roller bearings under given external radial and axial load. Teknisk Tidskrift, Mek., h.9, 1933.

[9] Rumbarger, J., Thrust bearing with eccentric loads. Mach. Des., February, 1962.

[10] Harris, T.A., Kotzalas, M.N., Rolling Bearing Analysis: Essential Concepts of Bearing Technology. Taylor \& Francis/CRC Press, 2007.

[11] Harris, T.A., Kotzalas, M.N., Rolling Bearing Analysis: Advanced Concepts of Bearing Technology. Taylor \& Francis/CRC Press, 2007. 